\title{
Ovarian leiomyoma with torsion: a case report
}

\author{
Pallipuram Seshadrinathan Bhageerathy ${ }^{1 *}$, Ajit Sebastian ${ }^{1}$, Dhanya Susan Thomas ${ }^{1}$, \\ Mayank Gupta ${ }^{2}$, Abraham Peedicayil ${ }^{1}$
}

\author{
${ }^{1}$ Department of Gynaecologic Oncology, Christian Medical College, Vellore, Tamil Nadu, India \\ ${ }^{2}$ Department of Pathology, Christian Medical College, Vellore, Tamil Nadu, India
}

Received: 17 October 2016

Revised: 19 October 2016

Accepted: 15 November 2016

\section{* Correspondence:}

Dr. Pallipuram Seshadrinathan Bhageerathy,

E-mail: drbhagee@gmail.com

Copyright: (C) the author(s), publisher and licensee Medip Academy. This is an open-access article distributed under the terms of the Creative Commons Attribution Non-Commercial License, which permits unrestricted non-commercial use, distribution, and reproduction in any medium, provided the original work is properly cited.

\begin{abstract}
We report an uncommon case of primary ovarian leiomyoma with torsion. Leiomyoma of the ovary is an extremely rare benign solid neoplasm of uncertain etiology. Our patient presented with abdominal pain and imaging showed right adnexal solid mass. All tumour markers except LDH were normal. The patient underwent laparotomy proceed right salpingo-ophorectomy. Intraoperatively, it was found to be twisted right ovarian solid tumour and histopathological examination revealed leiomyoma of ovary.
\end{abstract}

Keywords: Ovarian leiomyoma, Torsion

\section{INTRODUCTION}

Leiomyoma of the ovary is an extremely rare benign solid neoplasm which accounts for $0.5-1 \%$ of all benign ovarian tumours. ${ }^{1}$ Though several cases of primary ovarian leiomyomata are reported in literature, ours is probably the first reported case of ovarian leiomyoma with torsion. ${ }^{2}$

\section{CASE REPORT}

A 31year old Indian multipara presented to our outpatient department with history of lower abdominal pain. She experienced severe lower abdominal pain associated with vomiting 2 months ago when she was evaluated in a nearby hospital. An ultrasound scans of the abdomen reported as "probable mesenteric cyst". She was managed conservatively for 2 days after which the severity of the pain subsided, though she experienced dull aching type of lower abdominal pain off and on since then. There were no associated urinary or bowel symptoms.

Her menstrual cycles were regular once in every 28 days with normal flow for 3 days. She was married for 8 years with 2 children. Both were normal deliveries with last child birth 3 years ago after which she underwent abdominal puerperal tubal ligation.

There was no significant past medical or family history.

She was obese with a Body Mass Index of $32 \mathrm{~kg} / \mathrm{m}^{2}$. Abdominal examination revealed a $12 \times 10 \mathrm{~cm}$ firm mass with restricted mobility in the lower abdomen occupying predominantly the right iliac fossa, hypogastrium and the umbilical region. Bimanual examination showed a normal sized uterus which was pushed to the left side and a firm mass was felt through the right fornix.

Her CA 125 was 29.1 U/L and LDH was elevated to 996 U/L. Other germ cell tumour markers like alphafetoprotein and $\beta$ hCG were normal.

Ultrasound scan of the abdomen and pelvis showed a thick walled cystic lesion with large solid areas with dense echogenic foci and calcification as shown in Figure 1. There was no vascularity demonstrable within the mass. 
Magnetic resonance imaging of the abdomen and pelvis showed a large lobulated complex cystic mass measuring $14 \times 12 \times 7.5 \mathrm{~cm}$ arising from the right adnexa and located in the central portion of the abdomen. The mass had a T2 hypointense rim with a T1 hyperintense inner lining with heterogeneous contents. There was a funnel shaped extension inferiorly toward the uterine cornua with a suggestion of twisting of the pedicle. There was no ascites and other intraabdominal structures appeared normal.

She underwent laparotomy proceed Right salpingoophorectomy. There was about $200 \mathrm{ml}$ of blood stained ascitic fluid. Omentum was adherent to the anterior abdominal wall and the right adnexal mass. The right adnexal mass was firm and about $15 \times 15 \mathrm{~cm}$ in size and was adherent to the small bowel and omentum. It was twisted on its pedicle. Uterus was bulky and mobile. Left adnexa, peritoneal surfaces, diaphragm, liver, gall bladder, omentum, small and large bowel, appendix and mesentery appeared normal. Frozen section of the mass was reported as benign hence surgery stopped with right salpingo-ophorectomy. Her postoperative period was uneventful and she was discharged on the third postoperative day.

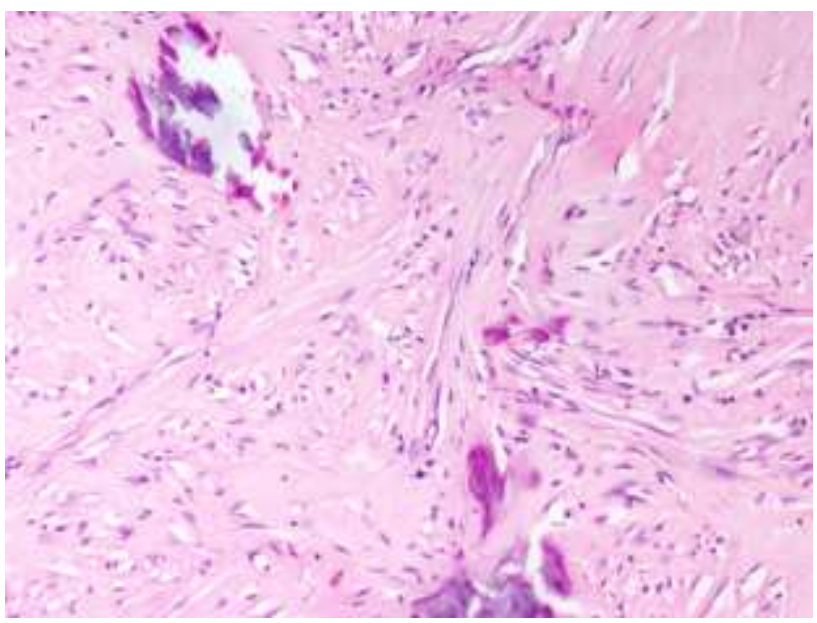

Figure 1: Ultrasound picture of right adnexal mass.

The histopathological examination of the excised ovarian mass revealed a circumscribed tumour displaying hyalinisation, infarction and foci of calcification. Bundles of benign smooth muscle cells were seen on microscopic examination (Figure 2) which was positive for Smooth Muscle Actin (SMA), but negative for h-Caldesmon. Chronic inflammatory cells were evident in the intervening areas. The features were favouring leiomyoma with extensive infarction, hyalinisation and calcification.

\section{DISCUSSION}

Primary ovarian leiomyoma is an extremely rare benign neoplasm of the ovary of uncertain etiology. Ovarian leiomyoma was first described by Sangli following which about 80 cases of the same are reported in literature. ${ }^{2}$ Most ovarian leiomyomas are small, usually less than 3 $\mathrm{cm}$ in diameter, asymptomatic, unilateral and found incidentally in premenopausal women ${ }^{3}$.Though our patient belonged to the reproductive age group and had unilateral ovarian tumour in line with the above description, she presented with abdominal pain and had a large mass. She was found to have twisted ovarian tumour which is probably the first reported case of torsion of ovarian leiomyoma.

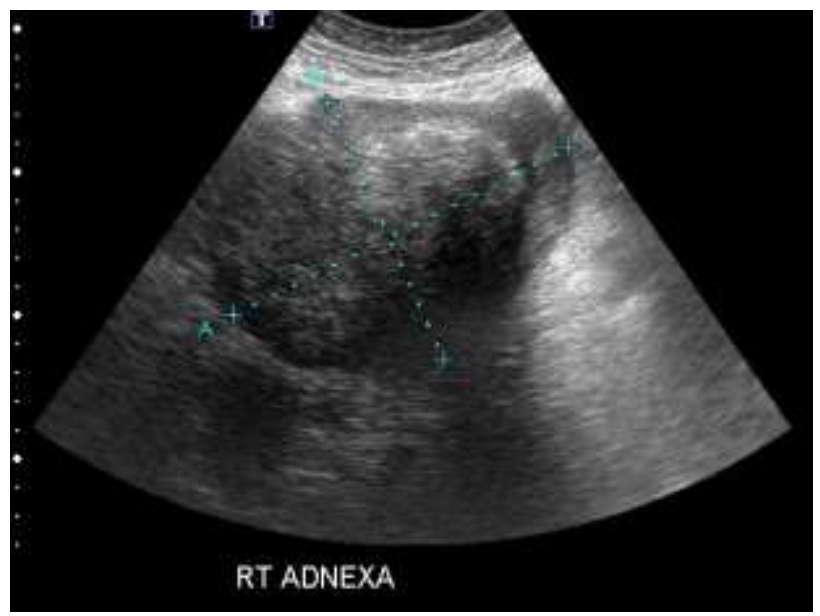

Figure 2: Haematoxylin and eosin (H\&E) stain showing fascicles of benign smooth muscle cells with intervening areas of hyalinization. Dystrophic calcification is evident; 10x magnification.

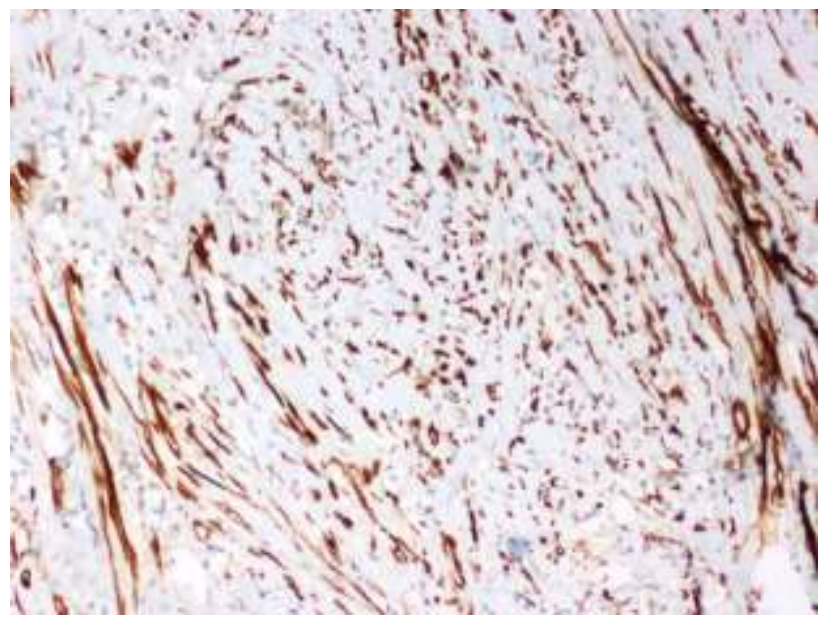

Figure 3: Tumour showing strong positivity for muscle specific actin, immunohistochemistry (IHC); 10x magnification.

Ovarian fibroids are often found in association with uterine fibroids suggesting common etiological factors though the exact pathogenesis is uncertain. They are thought to arise from the smooth muscle cells in the hilar blood vessels or in the ovarian ligament or from the multipotent cells in the ovarian stroma or the undifferentiated germ cells. ${ }^{2}$ There are reports of ovarian leiomyomas coexisting with endometriotic cyst, dermoid 
cyst and mucinous ovarian tumours which may be explained by smooth muscle metaplasia.

Due to the rarity of the condition, ovarian fibroids are not usually diagnosed preoperatively by history, clinical examination and imaging. The differential diagnoses include other solid tumours like fibroma, the coma, sclerosing stromal tumour or a broad ligament or subserous pedunculated fibroid. Leiomyosarcoma, gastrointestinal stromal tumours and spindle cell carcinoma should be ruled out in case of larger tumours.

The diagnosis is confirmed only by immuno histopathological examination of the surgical specimen. Immunohistochemistry shows diffuse positivity for SMA (Figure 3) and desmin in leiomyoma which is useful in the diagnosis. ${ }^{4} \alpha$-Inhibin can also be tested for confirmation which is positive in sex-cord stromal tumours and negative in leiomyomas. Mitotic activity, cytological atypia and tissue necrosis are looked into to differentiate it from leiomyosarcoma. h-Caldesmon is a sensitive and specific marker of smooth muscle differentiation and helps to diagnose Leiomyosarcoma. ${ }^{4}$

Ovarian leiomyoma typically has a benign course with excellent prognosis and recurrence is rare. ${ }^{2}$ Hence complete surgical excision of the tumour preserving normal ovarian tissue and fertility is advisable for young women. In those with associated multiple uterine fibroids and in elderly, total abdominal hysterectomy and bilateral salpingo-ophorectomy is preferred.

The patient's consent has been obtained.

Funding: No funding sources

Conflict of interest: None declared

Ethical approval: Not required

\section{REFERENCES}

1. Choi SY, Park JS, Lee JW, Kim BG. A Rare Case of Primary Ovarian Leiomyoma with Atypical Meig's Syndrome. Korean J Obstet Gynecol. 2012;55:285-9.

2. Gunasekaran I, Phansalkar M, Palo LB, Varghese RG. Ovarian Leiomyoma Along with Uterine Leiomyomata: A Common Tumour at an Uncommon Site. J Clin Diagn Res. 2015;9:ED01-2.

3. Taskin MI, Ozturk E, Yildirim F, Ozdemir N, Inceboz U. Primary Ovarian Leiomyoma: A case report. Int J Surg Case Rep. 2014;5:665-8.

4. Jena A, Patnayak R, Vijaylaxmi B, Lakshmi AY. Primary ovarian leiomyoma: An uncommon entityCase report and a brief review of literature. J Basic Clin Reprod Sci. 2013;2:54-6.

Cite this article as: Bhageerathy PS, Sebastian A, Thomas DS, Gupta M, Peedicayil A. Ovarian leiomyoma with torsion: a case report. Int J Reprod Contracept Obstet Gynecol 2017;6:295-7. 